\title{
Enhanced Fast Association for 802.15.4e-2012 DSME MAC Protocol
}

\author{
${ }^{1}$ Xuecheng Liu, ${ }^{1,2}$ Xiaoyun Li, ${ }^{1}$ Shijuan Su, ${ }^{1}$ Zhenke Fan, ${ }^{2,3}$ Gang Wang \\ ${ }^{1}$ Guangdong Provincial Key Laboratory of Robotics and Intelligent System, Shenzhen Institutes of Advanced Technology, \\ Chinese Academy of Sciences, Shenzhen, China \\ ${ }^{2}$ The Chinese University of Hong Kong, Hong Kong SAR, China \\ ${ }^{3}$ Center for Intelligent Sensors, Shenzhen Institutes of Advanced Technology, Chinese Academy of Sciences, \\ Shenzhen, China \\ e-mail: \{xuecheng.liu, xy.li, sj.su, zk.fan, gang.wang\}@siat.ac.cn \\ Xiaoyun Li (Correspondent author) Tel: 86-75586392142 Fax:86-75586392299
}

\begin{abstract}
IEEE 802.15.4e Deterministic \& Synchronous Multichannel Extension (DSME) mode was developed to meet the needs for industrial and commercial applications in large scale networks. However, long association delay is a critical problem in large scale networks due to serious collisions. This paper proposes an Enhanced Fast Association (EFastA) scheme in IEEE 802.15.4e DSME MAC protocol, in which a single Personal Area Network Coordinator (PANC) can associate numerous salve devices quickly with negligible collisions. Simulation results show that EFastA can associate hundreds of slave devices in 3 MDs (Multi-superframe Duration). The mean number of retransmissions required for a successful association is only $0.2 \%$ of that in standard DSME Fast Association (FastA) mode. The association convergence time of EFastA is $1.5 \% \sim 8.8 \%$ of that in FastA in the worst case, when MD = BI (Beacon Interval).
\end{abstract}

Keywords- IEEE 802.15.4e; DSME; Association; MAC;

\section{INTRODUCTION}

IEEE 802.15.4-2006 standard specified Wireless Medium Access Control (MAC) and Physical Layer (PHY) for LowRate Wireless Personal Area Networks (WPANs) [1]. The standard provides two modes of operation: the beaconenabled mode and non-beacon mode. The 802.15.4-2006 standard has attractive features such as energy-efficient communications, superframe structure, low duty-cycled, GTS and so forth. However, there are still several problems such as beacon collision problem and single channel operation. Besides, it cannot support large number of devices. The GTS described in IEEE 802.15.4-2006 can support applications requiring deterministic delay. However, there are still some limitations. Firstly, 802.15.4-2006 supports only up to seven guaranteed time slots (GTSs). Secondly, GTSs are supported only within one hop from the PAN Coordinator. Finally, GTSs are restricted to use a single channel [2].

Recently, IEEE 802.15 TG4 amended IEEE 802.15.42006 MAC specification. The MAC amendment mainly intended to support the industrial markets better. The new standard 802.15.4e-2012 has several major changes. It defines five new MAC operation modes: Deterministic \& Synchronous Multi-channel Extension mode (DSME), Low Latency Deterministic Network mode (LLDN), Time Slotted
Channel Hopping mode (TSCH), RFID Blink and Asynchronous multi-channel adaption (AMCA) [2].

Techniques for improving the performance of IEEE 802.15.4 association scheme for various applications have already been proposed by other authors. A new association scheme named fast association mechanism (FAM) for IEEE 802.15.4 real-time applications was proposed [3]. FAM improves the two steps of association: scanning through channels and initiating association. In the original standard, the scanning mechanism is not flexible. Application layer must wait until every channel is scanned even if the ideal PAN coordinator has been got in an earlier channel. FAM adds a new parameter named 'request type' in the MLMESCAN.request primitive, so it can actively terminate a scanning process.

Unlike DSME protocol [2] which requires a passive allocation of prospective devices, DFBS (Distributed Fast Beacon Scheduling) [4] allows an active allocation of prospective devices. After power on or reset, a node starts an active allocation request by broadcasting Active Association Request message and waiting for a reply. This active allocation request is performed over all channels until the node receives a reply.

In IEEE 802.15.4e, Fast association (FastA) is optional and not defined in IEEE 802.15.4-2006 [2]. The MAC behavior associated with FastA allows a device to associate in a reduced duration.

This paper is organized as follows. Section II presents an overview of IEEE 802.15.4e DSME MAC. Section III presents IEEE 802.15.4e association procedure. In Section IV, we propose the new association scheme: Enhanced Fast Association (EFastA) scheme. Section V presents simulation results and analysis. Finally, Section VI concludes the paper.

\section{OVERVIEW OF IEEE 802.15.4E DSME MAC WITH MUITI-SUPERFRAME STRUCTURE}

The DSME mode is designed for industrial applications, commercial applications and healthcare applications. These applications have the following requirements: deterministic latency, flexibility, high reliability, efficiency and so on. The DSME mode provides extension of number of GTS time slots and Multi-channel for capacity enhancement.

DSME-enabled network runs on beacon-enabled PAN. The Coordinator in a DSME-enabled PAN (PANC) shall 
periodically transmit an enhanced beacon to coordinator a multi-superframe structure. Multi-superframe structure is the extension of superframe structure defined in IEEE 802.15.4 MAC, but it can provide more than seven GTSs. As shown in Figure 1, a multi-superframe is a cycle of repeated superframes. The superframe duration can be divided into beacon period, contention access period (CAP), and contention free period (CFP). The multi-superframe structure is specified with the Beacon Order (BO), Multi-superframe Order (MO) and Superframe Order (SO). The beacon interval (BI), multi-superframe duration (MD) and superframe duration (SD) are related as follows:

$$
\begin{aligned}
& \mathrm{BI}=\text { aBaseSuperframeDuration } * 2^{\mathrm{BO}} \text { Symbols. } \\
& \mathrm{MD}=\text { aBaseSuperframeDuration } * 2^{\mathrm{MO}} \text { Symbols. } \\
& \mathrm{SD}=\text { aBaseSuperframeDuration } * 2^{\mathrm{SO}} \text { Symbols. }
\end{aligned}
$$

Within a beacon interval (BI), the multi-superframe duration will repeat $2^{(\mathrm{BO}-\mathrm{MO})}$ times. There are $2^{\text {(MO-SO) }}$ superframes in a multi-superframe. The number of devices supported in the DSME-enabled PAN is $2^{(\mathrm{MO}-\mathrm{SO})} * 7$, where 7 is the maximum number of devices to be allocated with GTS.

The superframe structure of the DSME MAC can be designed by specifying beacon interval, multi-superframe duration, superframe duration. If an industrial application requires that 20 devices are guaranteed to transmit every 2 $\mathrm{sec}$ with each transmission no less than $10 \mathrm{~ms}$, SO is firstly chosen as 4 to offer $15.36 \mathrm{~ms}$ slot and MO is chosen as 5 to offer 22 time slots, and $\mathrm{BO}$ is chosen as 7 to offer 1,966.08 ms beacon interval [5].

In order to save energy, the multi-superframe provides CAP reduction. When CAP reduction is enabled, the multisuperframe is illustrated in Figure 2. Only the first superframe (superframe ID 0) in the multi-superframe has the CAP and other superframes do not have a CAP. Thus provides more CFP.

In WPANs, where large number of sensor devices is densely deployed, mutual channel interference and channel fading will degrade throughput. DSME MAC provides two types of channel diversity schemes:channel hopping and channel adaptation to mitigate these channel impairments[6].

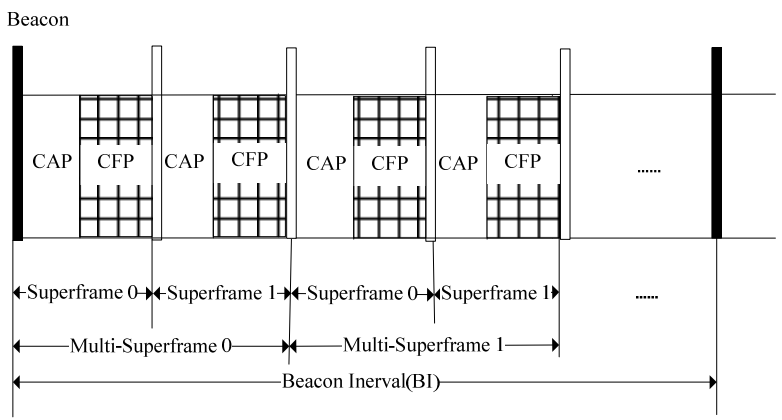

Figure 1. Multi-superframe Structure of DSME mode ( $\mathrm{SO}=4$, $\mathrm{MO}=5, \mathrm{BO}=10$ )

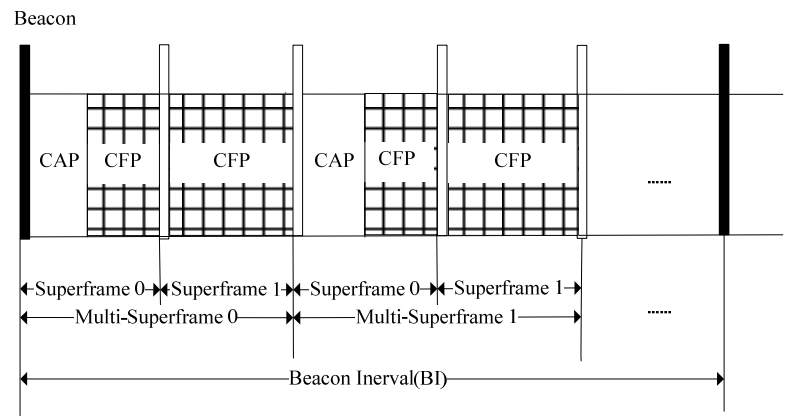

Figure 2. CAP reduction in DSME Multi-superframe Structure

\section{IEEE 802.15.4E NETWORK ASSOCIATION PROCEDURE}

\section{A. DSME device association}

Figure 3 illustrates a sequence of messages that may be used by a DSME device that is not tracking the beacon of the coordinator to successfully associate with a PANC. Due to low-energy considerations, the IEEE 802.15.4 association response command shall be sent to the device requesting association using indirect transmission. Indirect transmission leads to a significant delay in the association procedure. The device must wait macResponseWaitTime symbols which is equal to $32 * a B a s e S u p e r f r a m e D u r a t i o n$ (15.36ms in the $2.4 \mathrm{GHz}$ band). Therefore the device must wait $491.52 \mathrm{~ms}(32 * 15.36 \mathrm{~ms})$ in each initiating association. In practical applications, when more than 10 devices want to associate the PAN coordinator in a short period of time, overfull collisions will occur, results in association delay of tens of seconds [3].

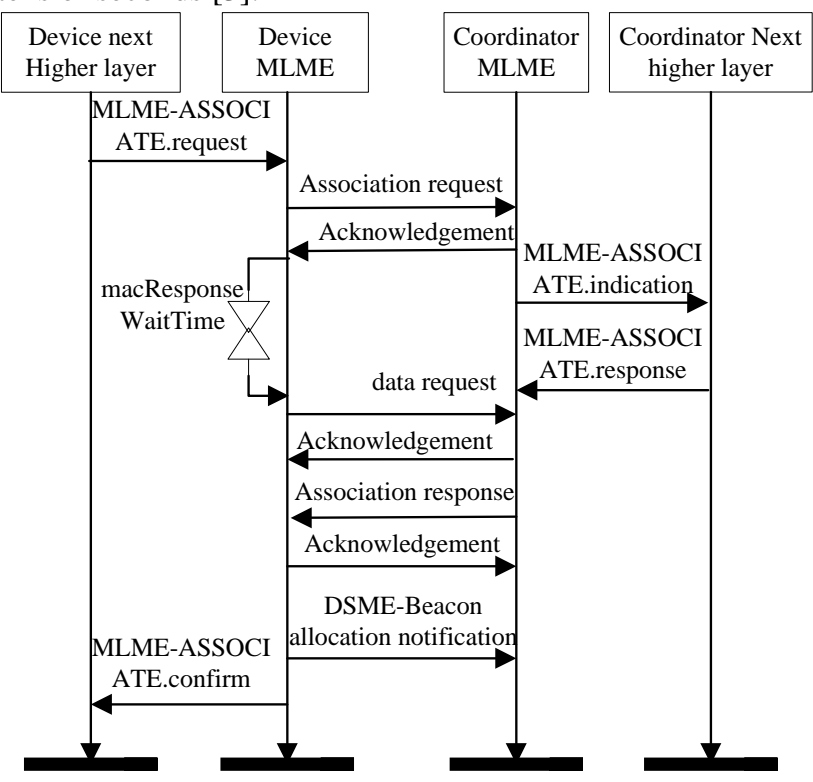

Figure 3. Message sequence chart for DSME device association 


\section{B. FAST Association Mechanism}

Fast association (FastA) is optional and defined in IEEE 802.15.4e-2012, but not defined in IEEE 802.15.4-2006.. FastA removes the fixed time-macResponseWaitTime, as illustrated in Figure 4, which reduces association delay.

The association request command allows a FastA device to request association with a PANC. If the PANC has sufficient resources, it allocates a short address to the device, and generates an association response command containing the short address and a status indicating a successful FastA. If the Association Type subfield of the Capability Information field is set to one, the PAN coordinator shall send the association response command frame to the devices directly. Otherwise, the association response command shall be sent as indirect transmission.

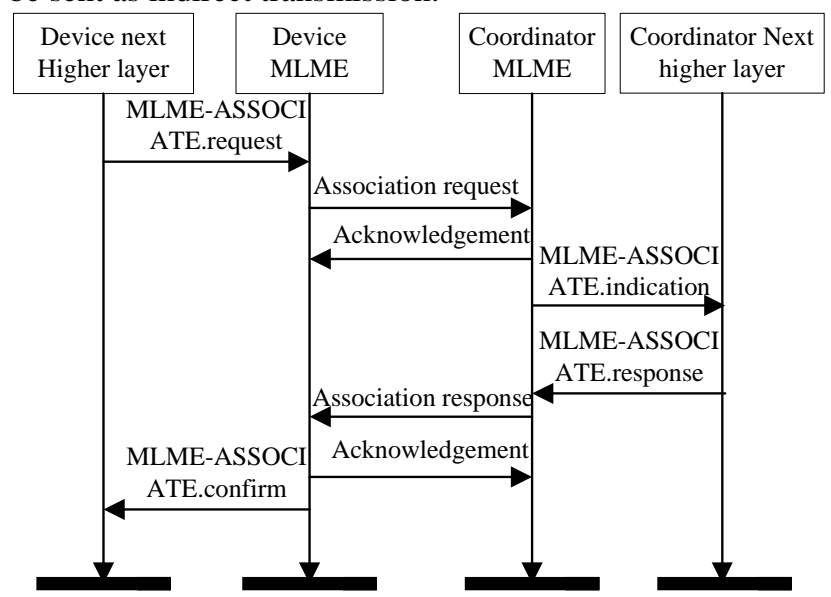

Figure 4.Message sequence chart of fast association

\section{ENHANCED FAST ASSOCIATION IN DSME}

\section{A. Scanning through Channels}

When a device wants to join a PAN, it must be associated with the PANC. The association procedure starts with a passive scan or an active scan or an enhanced active scan. The scan procedure allows a device to locate coordinator transmitting beacon frames. In active scan, a device will send beacon request command without listening to any beacon frame. In EFastA, active scan is not performed, because all devices shall be capable of performing passive scan across a specified list of channels. In addition, an FFD shall be able to perform energy detection (ED) and active scans. Besides, in low duty cycle networks, active scan leads to uncertain collisions. Simulation results shown that passive scan in EFastA is reliable and efficient for DSME mode.

\section{B. Association procedure}

In standard IEEE 802.15.4e DSME mode, when many unassociated devices independently perform a passive scan and locate a PANC simultaneously, they send fast association requests using CSMA/CA after receiving a beacon frame, resulting in a busy channel and severe collisions, as demonstrated by simulation in the next section.
To overcome such problems, EFastA provides the following association procedure. When a device $\mathrm{D}$ locates a PANC using passive scan, it will not send a fast association request immediately. Instead, $\mathrm{D}$ will select a random superframe ID, say in integer $\mathrm{L}(0<=\mathrm{L}<=\mathrm{K}-1$, where $\mathrm{K}=$ $\left.\mathrm{MD} / \mathrm{SD}, 2^{(\mathrm{MO}-\mathrm{SO})}\right)$. Then $\mathrm{D}$ will go to sleep and wake up when superframe $\mathrm{L}$ in the multi-superframe begins. $\mathrm{D}$ will then send a fast association request to PANC during superframe L's CAP period.

After receiving the EFastA request, the PANC will generates an association response command containing the short address and a status indicating success or failure. If the association fails, D repeats the association procedure and selects another random superframe ID until it associates with a PANC successfully. After that, D can request DSMEGTS(s) allocation, as defined by the standard. EFastA is compatible with standard IEEE 802.15.4e and FastA.

\section{Simulation And Result Analysis}

We use OMNeT++ network simulator to evaluate the performance of association of IEEE $802.15 .4 \mathrm{e}$ at the $2.4 \mathrm{GHz}$ frequency band. The performance of EFastA was evaluated in terms of network convergence time and mean number of collisions/retransmissions for association. Convergence time is the duration since unassociated device(s) received the first beacon frame until all devices have associated with PANC.

For large scale applications such as smart utility networks and process control application domains that have a large number of devices to be supported in a PAN, setting a relatively high value for macMultiSuperframeOrder can increase the number of devices supported by DSME-GTSs [2]. The parameters of superframe defined by DSME PANC are: $\mathrm{BO}=10, \mathrm{MO}=8, \mathrm{SO}=1, \mathrm{CAP}$ reduction is disabled. We adopt the beacon, slotted CSMA/CA transmission mode and channel transmission band-length is set to $250 \mathrm{Kpbs}$. Other main experimental parameters are set as follows: macMinBE=3, macMaxCSMABackoffs=4.

Initially, all the devices were unassociated, and were switched on to perform passive scan at the same time. The association procedure for both standard IEEE 802.15.4e DSME mode and EFastA mode can be found in Section III.

To ensure statistical reliability, 5 independent replications were run for each simulation with the same parameters before calculating the mean. Simulations for two different scenarios were carried out.

Scenario A. Enhanced Fast Association mode (EFastA): The multi-superframe structure of the PANC is shown in Figure 1. The maximum devices supported in the DSME-enabled PAN is $2^{(\mathrm{MO}-\mathrm{SO})} * 7=896(\mathrm{MO}=8, \mathrm{SO}=1)$, while CAP reduction is disabled. Initially the number of unassociated devices is 128, 256, 512 and 896 respectively, which means the saturation rate is $1 / 7,2 / 7,4 / 7,7 / 7$.

Scenario B. Standard IEEE 802.15.4e DSME FastA mode: When unassociated devices locate the PANC by receiving a beacon frame, they will send fast association 
requests using CSMA/CA immediately. The number of devices and other parameters are the same as in Scenario A.

Table 1 Simulation results for Scenario A

\begin{tabular}{|c|c|c|c|}
\hline Devices & $\begin{array}{c}\text { Retransm } \\
\text { ission }\end{array}$ & $\begin{array}{c}\text { CCA } \\
\text { Retry }\end{array}$ & $\begin{array}{c}\text { Converge } \\
\text { nce(MD) }\end{array}$ \\
\hline 128 & 0.09 & 3.0 & 1 \\
\hline 256 & 0.14 & 3.8 & 3 \\
\hline 512 & 0.28 & 4.8 & 3 \\
\hline 896 & 0.48 & 5.9 & 3 \\
\hline
\end{tabular}

Table 2 Simulation results for Scenario B

\begin{tabular}{|c|c|c|c|}
\hline Devices & $\begin{array}{c}\text { Retransm } \\
\text { ission }\end{array}$ & $\begin{array}{c}\text { CCA } \\
\text { Retry }\end{array}$ & $\begin{array}{c}\text { Converge } \\
\text { nce(BI) }\end{array}$ \\
\hline 128 & 12.5 & 53.0 & 15 \\
\hline 256 & 33.4 & 129.4 & 34 \\
\hline 512 & 119.6 & 397.2 & 103 \\
\hline 896 & 251.1 & 737.6 & 204 \\
\hline
\end{tabular}

Performance Comparison

Table 1 shows that in EFastA (Scenario A) the mean number of retransmissions required for a successful association is less than 0.5 , even when saturation rate is $100 \%$. Before sending an association request, a device D performs Clear Channel Assessment (CCA) using CSMA/CA as defined in standard [1]. If the channel is busy, D tries CCA again until a clear channel is detected. Then D sends its association request immediately. The mean number of CCA retries is less than 6 for all saturation rates. And the convergence time required for $100 \%$ of devices to associate with the PANC is no more than 3 MDs.

Table 2 shows that in standard IEEE 802.15.4e DSME FastA mode (Scenario $B$ ), the mean number of retransmissions is 12.5 for 128 associated devices. The mean number of retransmissions increases by approximately 2 to 3 times when the number of associated devices doubles. For 896 devices, there are on average 251.1 retransmission requests for a successful association, and the mean number of CCA retries for each device increases to 737.6.

In DSME MAC protocol, a PANC can associate with hundreds or thousands of slave devices. When EFastA is used, a device D can select a random superframe from the multi-superframe to send its association request, thus avoiding severe collisions and channel busy. In this way, a single PANC can support numerous devices while also avoiding network failure. In EFastA, the mean values of retransmissions and CCA retries for association are only $0.2 \%$ and $0.8 \%$ of that in normal fast association mode respectively - this holds for various numbers (128 to 896) of associated devices in Scenario B.

\section{CONCLUSION}

In this paper, we propose a new association scheme EFastA for IEEE 802.15.4e. Using EFastA, thousands of devices can quickly associate with the PANC with negligible collisions and retransmissions. Simulation results show that the convergence time to set up such a network is around 3 MDs for various numbers (128 to 896) of associated devices. The mean number of retransmissions for association is less than 0.5 . The association convergence time of EFastA is $1.5 \% \sim 8.8 \%$ of that in FastA in the worst case, when $\mathrm{MD}=\mathrm{BI}$. The convergence time and mean number of retransmissions are largely independent of the number of associated devices, indicating that enhanced mode is scalable to different network sizes.

The proposed enhanced FastA mode is suitable for applications on densely deployed networks where a powerful PANC can communicated with many energy-constrained slave devices such as Electronic Shelf Label system (ESLs). Future work will extend this concept to support heterogeneous networks.

\section{ACKNOWLEDGEMENTS}

This work was partly funded by Chinese NSFC research project No. 61271005, research projects KQC201109050096A and JC201005270368A of Shenzhen China, and Introduced Innovative R\&D Team of Guangdong Province "Robot and Intelligent Information Technology” (201001D0104648280).

\section{REFERENCES}

[1] IEEE Std. 802.15.4-2006, "Part 15.4: Wireless Medium Access Control (MAC) and Physical Layer (PHY) Specifications for LowRate Wireless Personal Area Networks (LR-WPANs)”, 2006.

[2] IEEE P802.15.4e-2012, "Part 15.4: Low-Rate Wireless Personal Area Networks (WPANs), Amendment 1: MAC sub-layer,” Feb. 2012.

[3] Fanliang Meng, Yigang Han "A New Association Scheme of IEEE 802.15.4 for Real-time Applications”, 5th International Conference on Wireless Communications, Networking and Mobile Computing, 2009. WiCom'09.

[4] Woon-yong Lee, Kwang-il Hwang, Young-Ae Jeon and Sang-Sung Choi, "Distributed Fast Beacon Scheduling for Mesh Networks", 2011 Eighth IEEE International Conference on Mobile Ad-Hoc and Sensor Systems

[5] Seong-Soon Joo, Bong-Soo Kim, Jong-Arm Jun, Cheol-Sig Pyo; "Enhanced MAC for the Bounded Access Delay", International Conference on Information and Communication Technology Convergence (ICTC), 2010

[6] Wun-Cheol Jeong, Junhee Lee, "Performance Evaluation of IEEE 802.15.4e DSME MAC Protocol for Wireless Sensor Networks” 2012 The First IEEE Workshop on Enabling Technologies for Smartphone and Innet of Things(ETSIoT) 